\title{
Age-Related Effects on Right Femoral Bone of Male Wistar Rats: A Morphometric and Biomechanical Study
}

\author{
Sheila Martins Puelker ${ }^{1}$ Sonia Regina Ribeiro de Castro ${ }^{1, \odot} \quad$ Romeu Rodrigues de Souza ${ }^{2}$ \\ Laura Beatriz Mesiano Maifrino ${ }^{3,4}, \odot$ Ricardo Aparecido Baptista Nucci ${ }^{1,5}$ Maria do Carmo Sitta
}

${ }^{1}$ Department of Aging Sciences, Sao Judas Tadeu University, Rua Taquari, Sao Paulo, Brazil

${ }^{2}$ Department of Anatomy, Nove de Julho University, Rua Vergueiro, Sao Paulo, Brazil

${ }^{3}$ Medical School of the ABC District, Av. Lauro Gomes, Sao Paulo, Brazil

${ }^{4}$ Dante Pazzanese Institute of Cardiology, Av. Dr. Dante Pazzanese, Sao Paulo, Brazil

${ }^{5}$ Department of Pathology, University of Sao Paulo Medical School, Sao Paulo, Brazil

${ }^{6}$ Division of Geriatrics, University of Sao Paulo Medical School, Sao Paulo, Brazil

Jealth Allied Sci ${ }^{\mathrm{Nu}}$ 2022;12:67-70.

\begin{abstract}
Address for correspondence Ricardo Aparecido Baptista Nucci, PhD, Department of Pathology, Av. Dr. Arnaldo, 455 Cerqueira Cesar, Sao Paulo 01246-903, Brazil (e-mail: nucci.ricardo.ab@gmail.com).
\end{abstract}

\begin{abstract}
Keywords

- aging

- biomechanics

- bone

Introduction Study of the variations of bone characteristics with age in different animal models is important to design musculoskeletal studies. Thus, this study aimed to evaluate the bone mass, dimensions, and biomechanical parameters of the femur in young, middle-aged, and aged Wistar rats.

Materials and Methods Thirty male rats (Rattus norvegicus) were divided in three groups ( $n=10$ per group)-3-month-old young rats, 12 -month-old middle-aged rats, and 18-months-old aged rats. The right femurs were subjected sequentially to morphometric study (bone weight, cortical thickness) and biomechanical tests (maximum resistance strength and bone stiffness).

Results We observed a significant increase in femur histological (cortical thickness) and biomechanical (maximum strength and bone stiffness) parameters with aging when compared with young animals.

Conclusions With the advancing age, the right femoral bone of middle-aged and old animals had greater variations when compared with young animals. However, further studies with the aid of a comparison between right and left femur and other long bones in both male and female rats are needed to corroborate with our findings.
\end{abstract}

\section{Introduction}

Bone tissue is a critical component to the function of the musculoskeletal system. ${ }^{1}$ The biomechanical characteristics of long bones, such as the bone density, maximum resistance force, and bone stiffness, differ between individuals, within the same individuals between bones, and within the same bone as a function of load distribution..$^{1-5}$ These characteristics are also dependent upon age and developmental stage. ${ }^{5,6}$ published online Jun 1, 2021
DOI https://doi.org/

10.1055/s-0041-1730107

ISSN $2582-4287$ (c) 2021. Nitte (Deemed to be University).

This is an open access article published by Thieme under the terms of the Creative Commons Attribution-NonDerivative-NonCommercial-License, permitting copying and reproduction so long as the original work is given appropriate credit. Contents may not be used for commercial purposes, or adapted, remixed, transformed or built upon. (https://creativecommons.org/licenses/by-nc-nd/4.0/).

Thieme Medical and Scientific Publishers Pvt. Ltd. A-12, 2nd Floor,

Sector 2, Noida-201301 UP, India 
Although several studies have been performed to examine the effects of growing and aging on bone biomechanical properties, these studies have involved comparison of young and adult individuals. ${ }^{3,7}$ However, few data on age-related changes in histological and biomechanical parameters of Wistar rats are available. Such data are important for studies of the response of the bone experimental stimuli in aged rats to distinguish the response to the stimuli from accompanying age-related changes. . $^{8-10}$

The purpose of the present work was to investigate the bone density due to its weight, maximum resistance force, and bone stiffness of the Wistar rat right femur, as a function of growing and aging.

\section{Materials and Methods}

\section{Animals}

The institutional animal care and use committee of the university approved the experimental protocol and all procedures were in accordance with the guiding principles of the National Institute of Health Guide for the Care and Use of Laboratory Animals (protocol 011/20). The animals used in the present study were male Wistar rats (Rattus norvegicus) purchased from the Laboratory Animal Center of Sao Judas Tadeu University, Sao Paulo, Brazil. Thirty animals were divided in three groups ( $n=10$ per group): (a) 3-month-old young rats, (b) 12-month-old middle-aged rats, and (c) 18-months-old aged rats. During the experiment, standard chow and water were provided ad libitum to all animals. Rats were weighed before being euthanized with pentobarbital intraperitoneally. Then, the right hind limb was dissected from the body. After removal of all muscles and other tissues, the femur was then weighed and prepared for analyses.

\section{Measurement of Cortical Thickness}

All bones were fixed in 100\% ethanol, decalcified, embedded in methacrylate, and sectioned into histological sections with a tungsten knife (Junk, Carl Zeiss, Germany). To determine the cortical thickness, histological sections stained with $0.1 \%$ toluidine blue ( $\mathrm{pH}$ 6.4) were submitted to a digital image analysis program (AxioVision, Carl Zeiss, Germany) with 100× magnification in a microscope (NIKON, LABOPHOT-2A) with millimeter scale reticle, calibrated using a Vernier scale. A total of 200 images were examined per group, that is, 20 images per animal. The cortical thickness of the longitudinal sections was measured at four equidistant points of the distal extremity of the femur. This method was previously described for bone and cartilage evaluation. ${ }^{11}$ The final value analyzed was the average of these points expressed in micrometers $(\mu \mathrm{m})$.

\section{Biomechanical Tests}

Measurements were taken on a KRATOS universal biomechanical testing machine, model K5002, ${ }^{12}$ and all tests were documented by a 790 BBC Goerz Metrawatt Servogor graphic recorder to obtain the "load-deformation" curve of each test. ${ }^{13}$ A three-point bending test device was used, where a 7 -mm diameter $\times 30$-mm wide cylindrical cutter is attached to the crosshead of the testing machine, with a load cell of $100 \mathrm{~kg}$-force (kgf), to a scale of two, therefore with $25 \mathrm{kgf}$. Two 4-mm diameter metal cylinders were used to hold the bones in position, adjusted with a bench vise mounted to the base of the machine with a $25-\mathrm{mm}$ distance between the supports, with the cutter centered.

The bone was positioned and centered on the cylindrical supports by attaching the proximal portion of the femur between the neck and the lesser trochanter. It was observed that, in that position, the bone remained stationary during the test, with no displacements that could compromise the test. The cutter attached to the load cell was directed perpendicular to the axis of the femoral diaphysis and moved at a constant speed of $5 \mathrm{~mm} / \mathrm{min}$ until bone fracture occurred.

The maximum resistance strength and deformation values were determined for each curve. The following parameters were considered: (1) Maximum resistance strength in newton (N) which represents the maximum load applied to the femur until fracture occurs; it was used to calculate the bone stiffness. (2) Deformation in millimeters ( $\mathrm{mm}$ ) which is a measure of the deformation (flexural strain) suffered by the bone at the maximum load limit. (3) Bone stiffness in kilonewtons/millimeter $(\mathrm{kN} / \mathrm{mm})$ which is calculated by dividing force $(\mathrm{kN})$ by deformation $(\mathrm{mm})$. This parameter represents the deformability of the material when the load is applied and, therefore, represents the stiffness of the material.
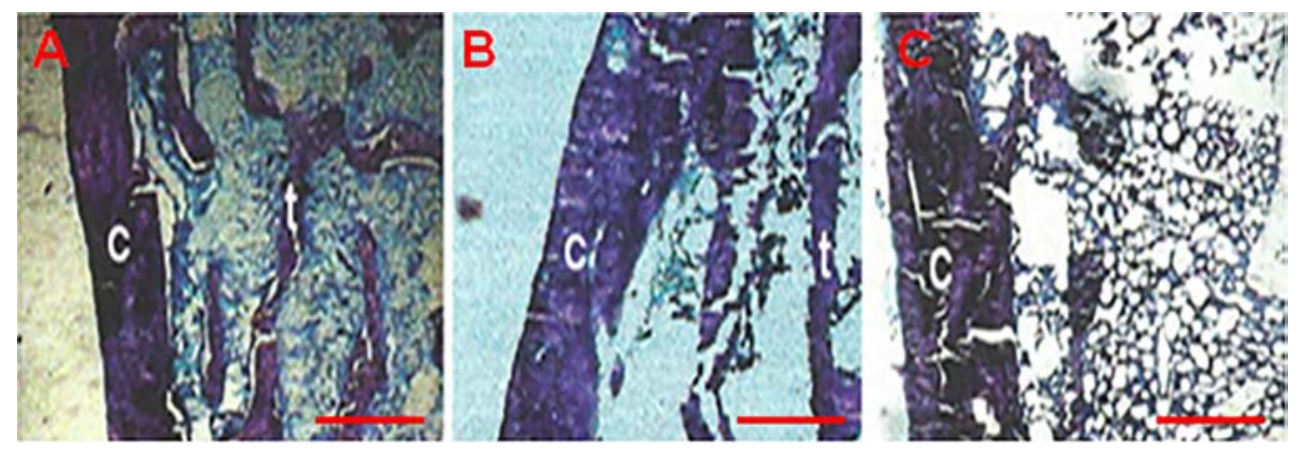

Fig. 1 Photomicrographs of histological sections obtained from the distal extremity of the femur for measurement of the cortical thickness (C) in toluidine blue staining. The trabeculae of spongy bone ( $\mathrm{t}$ ) is evidenced in the images. The (A) 3-month-old group, (B) 12-month-old group, and (C) 18-month-old group. Scale bar $=200 \mu \mathrm{m}$. 


\section{Statistical Analysis}

Data were expressed as mean \pm standard deviation (SD). We conducted a one-way analysis of variance (ANOVA) with Newman-Keuls posttest in GraphPad Prism 5.0 software (GraphPad Prism, Inc., San Diego, California). The $\alpha$ level was set at the 0.05 .

\section{Results}

- Table 1 shows that body weight significantly increased $50.38 \%$ and $42.33 \%$ in 12-month-old and 18-month-old animals, respectively, when compared with 3-month-old rats. Additionally, bone weight significantly increased $38.06 \%$ and $35.73 \%$ in 12 -month-old and 18-month-old rats, respectively, when compared with the group with 3-month-old rats. In regard to cortical thickness, we observed a significantly increase of $25.59 \%$ and $27.77 \%$ in 12-month-old and 18-month-old rats, respectively, when compared with the group with 3-month-old rats. Representative photomicrographs of the cortical histology between the groups are seen in Fig. 1.

Biomechanical analysis showed a sharp increase of $81.15 \%$ and $82.39 \%$ of maximum strength in 12 -month-old and 18-month-old rats, respectively, when compared with 3-month-old rats (-Table 1). Finally, we observed that stiffness had a sharp increase of $136.28 \%$ and $102.02 \%$ in 12-month-old and 18-month-old rats, respectively, when compared with 3-month-old rats.

\section{Discussion}

Knowledge of the bone aging is important to understand common pathological processes affecting the elderly. Thus, our study analyzed the effects of aging on the femoral cortical thickness, maximum strength, and stiffness of Wistar rats for the design of future gerontological studies.

In the first 7 months of life animals have a constant weight gain that gradually tend to a relative stability, which is observed in our results regarding body and bone weight, and cortical thickness, as this parameters is influenced by weight. This phenomenon is due to the increased volume of tissues and organs that occurs during growth and aging. ${ }^{14,15}$ In addition, we suggest that our results related to the cortical thickness increased in aging is related to the "cortical drift" phenomenon. ${ }^{9}$ Cortical drift occurs rapidly during prepubertal growth, levels off after closure of the epiphyseal plate, and increases again in the elderly, often resulting in weaker bone, due to increased porosity, with a wider diameter and significantly thinner cortices. ${ }^{9,14}$ However, cortical drift does not occur uniformly around the bone diameter. Thus, in our study we analyzed equidistant points ${ }^{11}$ to measure cortical thickness in the bone heterogeneity.

In this study, biomechanical properties were assessed using force, which is a parameter that measures bone strength, and using stiffness, which measures the deformation of the material when maximum force is applied. The 12-month-old and 18-month-old groups showed a significant increase in maximum strength in relation to young animals. Maximum strength is a common parameter used on experimental osteoporosis. ${ }^{16}$ Stiffness in the aged groups showed a significant difference in relation to the initial 3-month-old group. As for aging, a decrease of bone strength and increase in bone stiffness is expected. ${ }^{9}$ However, as our animals were kept healthy, microcracks may not have happened as much as seen in pathological conditions (e.g., osteoporosis), as this is a mechanism related to the decrease of bone strength due to the disruption of both bone mineral crystallites and collagen fibrils, which may explain the increased maximum strength across age in our study. ${ }^{9,14}$

However, our results should be examined considering the study limitations. We did not analyze other long bones (e.g., tibia and fibula) or the left femoral bone to compare the difference between right and left bones in the animals. However, our results are in accordance with the literature. ${ }^{9,14}$ Additionally, further studies should include female rats in the analysis as estrogen deprivation due to the aging process is associated with decreased bone mineral density. ${ }^{17}$ Although our study has limitations, we also have some advantages. We presented histological and biomechanical parameters of the effect of aging on the femoral bone using techniques previous described..$^{11}$ Additionally, the aged groups maintained normal spontaneous movement inside the cage and, therefore, serves as a model of normal bone aging for laboratory rats under standard conditions.

\section{Conclusion}

We suggest that aging is a key factor in increasing bone weight, cortical thickness, maximum resistance strength, and bone stiffness in Wistar rats. Thus, we hope that these

Table 1 Body and bone weight, cortical thickness, maximum strength, and stiffness of the femur in 3-month-old, 12-month-old, and 18-month-old groups ( $\mathrm{n}=10$ per group)

\begin{tabular}{|l|l|l|l|}
\hline Parameters & 3-month-old rats & 12-month-old rats & 18-month-old rats \\
\hline Body weight $(g)$ & $274.50 \pm 5.88$ & $412.80 \pm 14.00^{\mathrm{a}}$ & $390.70 \pm 13.48^{\mathrm{ab}}$ \\
\hline Bone weight $(\mathrm{g})$ & $0.5964 \pm 0.05$ & $0.8234 \pm 0.09^{\mathrm{a}}$ & $0.8095 \pm 0.08^{\mathrm{a}}$ \\
\hline Cortical thickness $(\mu \mathrm{m})$ & $380.20 \pm 9.99$ & $477.50 \pm 20.50^{\mathrm{a}}$ & $485.80 \pm 17.49^{\mathrm{a}}$ \\
\hline Maximum strength $(\mathrm{N})$ & $72.59 \pm 3.57$ & $131.50 \pm 5.50^{\mathrm{a}}$ & $132.40 \pm 7.17^{\mathrm{a}}$ \\
\hline Stiffness $(\mathrm{kN} / \mathrm{m})$ & $79.99 \pm 4.44$ & $189.00 \pm 12.50^{\mathrm{a}}$ & $161.60 \pm 8.02^{\mathrm{ab}}$ \\
\hline
\end{tabular}

Data expressed as mean \pm standard deviation (SD).

${ }^{a} P<0.05$ versus 3 -month-old rats.

${ }^{\mathrm{b}} P<0.05$ versus 12 -month-old rats. 
parameters may encourage future musculoskeletal studies using Wistar rats as an experimental model of aging.

\section{Note}

The institutional animal care and use Committee of the university approved the experimental protocol and all procedures were in accordance with the guiding principles of the National Institute of Health Guide for the Care and Use of Laboratory Animals (protocol 011/20). The animals used in the present study were male Wistar rats (Rattus norvegicus) purchased from the Laboratory Animal Center of Sao Judas Tadeu University, Sao Paulo, Brazil.

\section{Authors' Contributions}

S.M.P. and S.R.R.C. contributed to investigation, writing, and review and editing. R.R.S. contributed to conceptualization, project administration, and writing (original draft). L.B.M.M. contributed to methodology, resources, supervision, validation, and writing-review and editing. R.A.B.N. contributed to data curation, formal analysis, visualization, and writing-original draft. M.C.S. contributed to formal analysis, supervision, and writing-review and editing.

\section{Conflict of Interest}

None declared.

\section{References}

1 Rittweger J, Beller G, Ehrig J, et al. Bone-muscle strength indices for the human lower leg. Bone 2000;27(2):319-326

2 Hagihara Y, Nakajima A, Fukuda S, Goto S, Iida H, Yamazaki M. Running exercise for short duration increases bone mineral density of loaded long bones in young growing rats. Tohoku J Exp Med 2009;219(2):139-143

3 Iwamoto J, Takeda T, Sato Y. Effect of treadmill exercise on bone mass in female rats. Exp Anim 2005;54(1):1-6
4 Viboolvorakul S, Niimi H, Wongeak-in N, Eksakulkla S, Patumraj S. Increased capillary vascularity in the femur of aged rats by exercise training. Microvasc Res 2009;78(3):459-463

5 Sherk VD, Rosen CJ. Senescent and apoptotic osteocytes and aging: exercise to the rescue? Bone 2019;121:255-258

6 Zaidi M, Lizneva D, Kim SM, et al. FSH, bone mass, body fat, and biological aging. Endocrinology 2018;159(10):3503-3514

7 Stenderup K, Justesen J, Clausen C, Kassem M. Aging is associated with decreased maximal life span and accelerated senescence of bone marrow stromal cells. Bone 2003;33(6):919-926

8 Chartier SR, Mitchell SAT, Majuta LA, Mantyh PW. The changing sensory and sympathetic innervation of the young, adult and aging mouse femur. Neuroscience 2018;387:178-190

9 Boskey AL, Coleman R. Aging and bone. J Dent Res 2010;89(12):1333-1348

10 Ferrucci L, Baroni M, Ranchelli A, et al. Interaction between bone and muscle in older persons with mobility limitations. Curr Pharm Des 2014;20(19):3178-3197

11 Calderon MF, Nucci RAB, de Souza RR. Age-related changes in the articular cartilage of the mandible of rats. Acta Histochem 2020;122(2):151501

12 Dota AF, Zenaide MR, Demange MK, Camanho GL, Hernandez AJ. Study of the mechanical properties of the posterior cruciate ligament and patellar tendon on fresh human cadavers after radiofrequency shrinkage. Acta Ortop Bras 2007;15:138-142

13 Bolliger Neto R, Rossi JD, Leivas TP. Experimental determination of bone cortex holding power of orthopedic screw. Rev Hosp ClinFac Med Sao Paulo 1999;54(6):181-186

14 Curtis E, Litwic A, Cooper C, Dennison E. Determinants of muscle and bone aging. J Cell Physiol 2015;230(11):2618-2625

15 Pando R, Masarwi M, Shtaif B, et al. Bone quality is affected by food restriction and by nutrition-induced catch-up growth. J Endocrinol 2014;223(3):227-239

16 Rubin CD. Emerging concepts in osteoporosis and bone strength. Curr Med Res Opin 2005;21(7):1049-1056

17 Hernandez CJ, Beaupré GS, Carter DR. A theoretical analysis of the relative influences of peak BMD, age-related bone loss and menopause on the development of osteoporosis. Osteoporos Int 2003;14(10):843-847 